Research Article

\title{
Efficacy of terbutaline in preterm labour - our experience
}

\author{
Priyadarshini Bai G. ${ }^{1 *}$, Ravi Kumar P. ${ }^{2}$, T. N. Kumar ${ }^{1}$
}

\author{
${ }^{1}$ Department of Pharmacology, \\ ${ }^{2}$ Department of Pulmonology, \\ Sri Siddhartha Medical College, \\ Tumakuru, Karnataka, India \\ Received: 14 July 2016 \\ Accepted: 13 August 2016

\section{*Correspondence to: Dr. Priyadarshini Bai G., Email: ravi_darshini@ yahoo.co.in}

Copyright: (C) the author(s), publisher and licensee Medip Academy. This is an openaccess article distributed under the terms of the Creative Commons Attribution NonCommercial License, which permits unrestricted noncommercial use, distribution, and reproduction in any medium, provided the original work is properly cited.

\begin{abstract}
Background: Preterm birth is one of the important causes of perinatal mortality and morbidity in developed countries after congenital malformations. Postponement of delivery plays essential role in preventing such incidences by allowing fetal lung maturity.

Methods: This is a prospective study conducted over a period of 20 months among the patients admitted for preterm labour with gestational period between 27 to 36 weeks. After the patients obeyed all the inclusion criteria, they were administered terbutaline $250 \mu \mathrm{g}$ subcutaneously every 8th hourly followed by 5 mg tablet orally twice daily till contractions ceased. The patients were analysed for gestational period at which they presented with preterm labour contractions, associated risk factors, response to terbutaline, and gestational period at delivery, perinatal complications, neonatal and maternal outcomes. The data obtained were analysed by descriptive analysis.
\end{abstract}

Results: There were 1,678 deliveries during study period, out of which 207 (12\%) presented with preterm labour and $20(\sim 10 \%)$ patients received terbutaline. Idiopathic (50\%) and vaginal infections (25\%) were common causes of preterm labour among the patients who received terbutaline. The labour was prolonged by 3 - 5 days among 8 patients, 7 - 15 days in 9 patients and 15 - 30 days in 3 patients. Common maternal side effects were nausea and tremors. Neonatal outcome was good in most of the cases except in $5(25 \%)$ neonates who required NICU admission but they all recovered well without any morbidity.

Conclusions: Administration of terbutaline prolonged labour without any serious maternal complications and better neonatal outcome as $90 \%$ of the neonates were born without any complications. Hence, terbutaline can be used as a safe and effective tocolytic agent among patients presenting with preterm labour.

Keywords: Preterm labour, Terbutaline, Tocolytic, Efficacy, Safety,

\section{INTRODUCTION}

According to the World Health Organization (WHO) recommendation, preterm labor is defined as labor starting earlier than 37 completed weeks (less than 259 days) from the first day of the last menstrual period. ${ }^{1}$ Preterm delivery affects approximately one in 10 births and is the cause of at least $75 \%$ of neonatal deaths, excluding those related to congenital malformations. Although the cause of preterm labour is unknown, the rate of fetal morbidity can be reduced with early and accurate diagnosis of preterm labour, intervention to delay preterm delivery, administration of corticosteroids and provision for neonatal care. ${ }^{2}$
Preterm birth is the single most important cause of perinatal mortality and morbidity in developed countries. About 13 million preterm births are reported worldwide annually. ${ }^{3}$ Incidence of preterm labor in India is $23.3 \%$ and of preterm delivery is $10-69 \% .^{4}$

The etiology of preterm is multifactorial. Four different pathways have been identified that can result in preterm birth and have considerable evidence: premature fetal endocrine activation, uterine over distension, decidual bleeding and intrauterine inflammation/infection. From a practical point a number of factors have been identified that are associated with preterm labour: Incompetency of cervix, multiple pregnancies, Abnormality of uterus, Chorioamnionitis, infection outside uterus, Placental 
abnormalities like abruption placenta, circumvallate placenta.

Though many pharmacological drugs are available to supplement the treatment in preterm labour, there is still a debate as to which of these drugs is the best. As NICU care has improved over the last decade, viability has reduced to approximately 30 weeks in the Indian setup. Though tocolytics may not help in prolonging pregnancy to such an extent that fetus may grow and mature further, it may defer delivery sufficiently to allow the mother to be brought to a specialized centre that is equipped and staffed to handle preterm deliveries. ${ }^{5}$

Use of more selective tocolytic therapy offers hope that new therapeutic approach may increase rates of fetal survival. Among most effective tocolytic agents used in the management of preterm birth are - $\beta 2$ agonists, calcium channel blockers, prostaglandin synthetase inhibitors, nitric oxide donors and oxytocin receptor antagonists. ${ }^{6}$ However, none have been found to be completely effective, and the choice of drug is often limited by adverse effects. ${ }^{7,8}$

Beta-adrenergic agonists are among the most commonly used tocolytic agents. It has been shown that these agents postpone the delivery for 24,48 hours and even 7 days. ${ }^{9,10}$ Postponement of pregnancy for at least 48 hours allows the corticosteroids to reach their maximum effect for reducing respiratory distress syndrome and its sequelae. $^{11}$

\section{METHODS}

This study was conducted over a period of 20 months. Women who were aged between 19 to 35 years with gestational age ranging from 27 weeks to 36 weeks were recruited into the study. The gestational age was based on the last menstrual period and a reliable menstrual history and/or an ultrasound before 20 weeks of gestational age. Any women should not have been administered with tocolytics at least for the last 7 days at the initiation of the study. The study was conducted after the approval obtained from the institutional ethical committee. The study protocol and its benefits and complications were explained to all participants, and all recruited patients signed the informed consent form after completely understanding the study protocol explained to them in their local language.

Preterm labour was diagnosed on the basis of regular objective uterine contractions. The contractions should appear at least once every 10 minutes, lasting for 30-60 seconds with cervical dilatation less than $3 \mathrm{cms}$ and associated with cervical changes (effacement and dilatation). Exclusion criteria included any co-existing conditions like severe pre-eclampsia, eclampsia, diabetes mellitus, cardiovascular diseases, hyperthyroidism, a clinical diagnosis of abruption placentae, documented intrauterine infections and cervical dilatation of more than $4 \mathrm{cms}$, fetal distress and severe fetal growth restriction.

Each woman was investigated for hemoglobin estimation, albuminuria, glycosuria, bacteraemia, blood group estimation, and random blood sugar. Electrolytes and total and differential leukocyte count were estimated. High vaginal swabs were taken. Their follow up was done till delivery. A special note of any maternal or fetal complications was made. For all of these women, appropriate intra-partum and postpartum care were given to mother as well as the baby. Thereafter comparison of each drug in relation to maternal and fetal side effects and prolongation of pregnancy was done.

Terbutaline is one of several preterm labor drugs currently being administered to pregnant women to control preterm labour. It is used for short time period (typically 48-72 hours) as an emergency measure to halt preterm labor for women at high risk of preterm labor. When used for short term periods it is initiated with a dose of $10 \mathrm{mcg} / \mathrm{min}$ and is increased by $5 \mathrm{mcg} / \mathrm{min}$ every $10 \mathrm{~min}$ until uterine contractions cease or $0.25 \mathrm{mg}$ subcutaneously and repeated hourly until contractions stop, after which maintenance dose is decreased by $5 \mathrm{mcg}$ every $30 \mathrm{~min}$ to the lowest effective dose. After the suppression of uterine contractions, oral dose of 15 $\mathrm{mg}$ /day is given and continued until 36 weeks as a maintenance dose. Side effects were recorded during this period. Two doses of Betamethasone were given $12 \mathrm{mg}$ IM every 24 hours for maturation of fetal lung. Antibiotics were given in case of premature rupture of membranes. Fetal heart rate and uterine contractions, as well as maternal blood pressure and pulse rate were assessed every 12 hours.

Prolongation of pregnancy for a period of 48 hours was the primary outcome in this study. Patients whose contractions discontinued after 48 hours were discharged from the hospital with the maintenance therapy. If uterine contractions reappeared, relapse was diagnosed and treatment was repeated as indicated above. Medication was continued until 37 weeks of gestation unless no side effects appeared. Neonatal weight, APGAR score were recorded after delivery. Neonatal complications such as haemorrhage or infections were also recorded. The secondary outcome of the study was the length of prolongation of pregnancy and the side effects of the medication. The data obtained were analysed by using descriptive analysis.

\section{RESULTS}

There were 1,678 deliveries during study period, out of which $207(12 \%)$ presented with preterm labour and 20 $(\sim 10 \%)$ patients received terbutaline. The most common risk factor for preterm labour in our study was idiopathic $(50 \%)$ and vaginal infection $(25 \%)$ which was followed by $5 \%$ women each with cervical incompetence, polyhydromnios, PROM, previous history of preterm 
labour and multiple pregnancies. This is depicted in Figure 1.

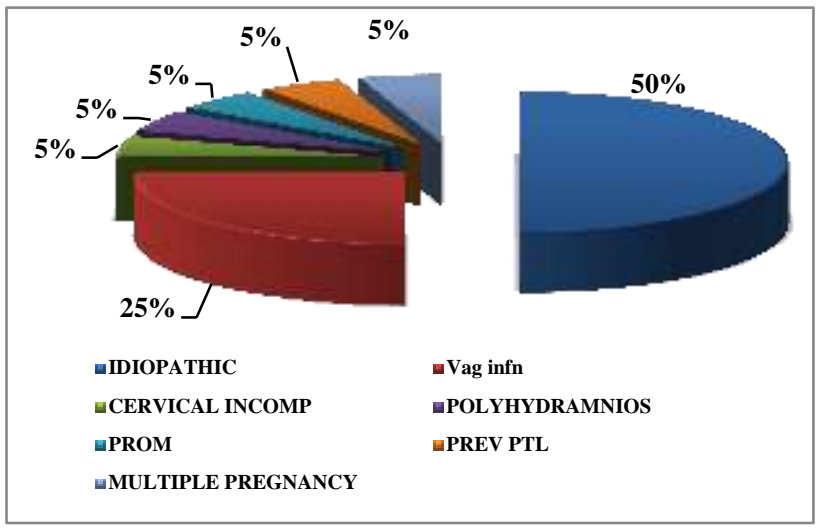

Figure 1: Risk factors.

The most common risk factor for preterm labour in our study was idiopathic (50\%) and vaginal infection $(25 \%)$ which was followed by $5 \%$ women each with cervical incompetence, polyhydromnios, PROM, previous history of preterm labour and multiple pregnancies.

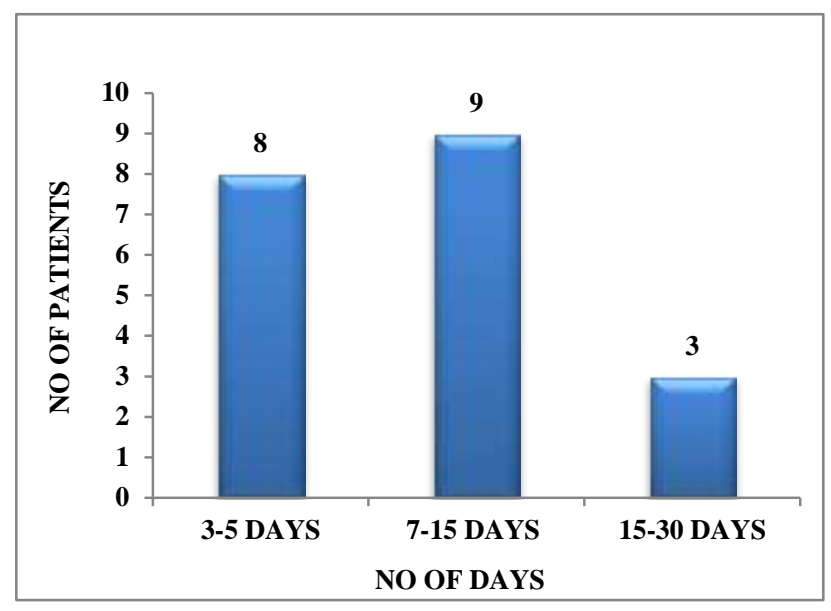

Figure 2: Number of days of prolongation of labour.

Labour was prolonged for 3 - 5 days in 8 (40\%) women; 7 - 15 days in 9 women (45\%) and 15 - 30 days in 3 women $(15 \%)$.

Prolongation of labour, which is the main efficacy point in our study, is shown in Figure 2. Labour was prolonged for 3 - 5 days in 8 (40\%) women; 7 - 15 days in 9 women $(45 \%)$ and 15 - 30 days in 3 women (15\%).

The mode of delivery is shown in Figure 3. Out of 20 women, $12(60 \%)$ of them delivered through normal vaginal delivery, 7 (35\%) women underwent caesarian section and $1(5 \%)$ woman required forceps delivery.

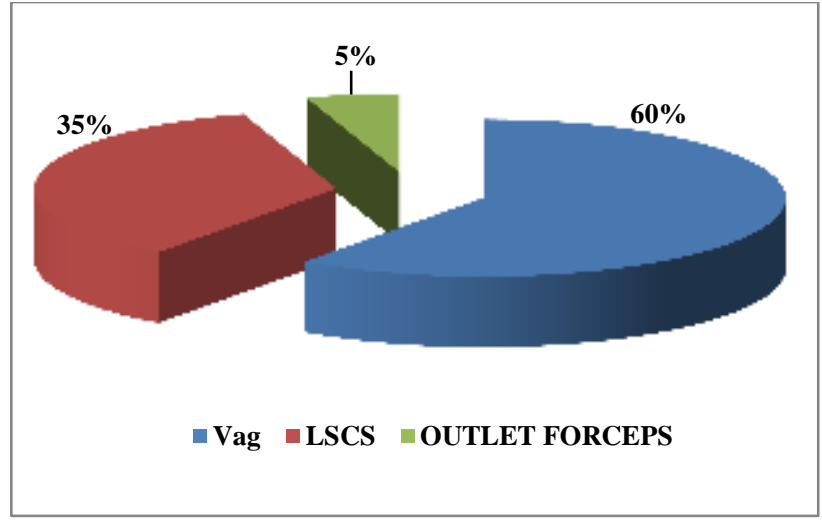

Figure 3: Type of delivery.

Out of 20 women, $12(60 \%)$ of them delivered through normal vaginal delivery, $7(35 \%)$ women underwent caesarian section and 1 (5\%) woman required forceps delivery.

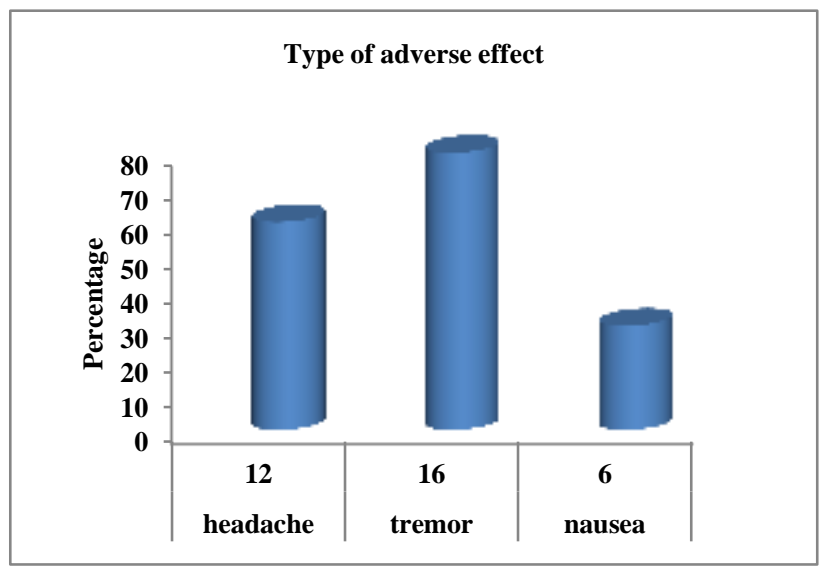

Figure 4: Maternal side effects.

Common maternal side effects were tremors (80\%), headache $(60 \%)$ and nausea $(30 \%)$.

Common maternal side effects were tremors (80\%), headache $(60 \%)$ and nausea $(30 \%)$ as shown in Figure 4.

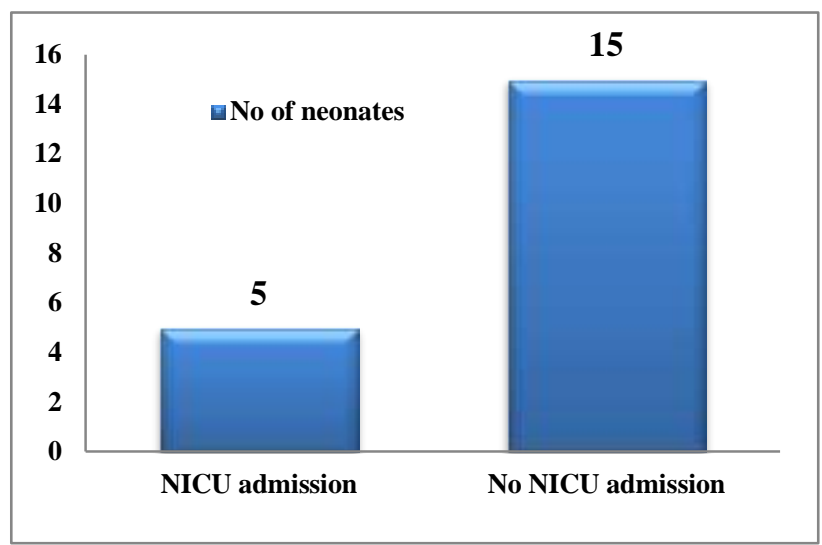

Figure 5: Number of NICU admissions. 
Figure 5 shows number of neonates admitted to NICU for monitoring after the birth. In our study only $5(25 \%)$ neonates required NICU admission as they were preterm deliveries. However the overall neonatal outcome was good without any morbidity and mortality.

\section{DISCUSSION}

Preterm labour is a major contributor to perinatal mortality and morbidity and affects $6-7 \%$ of births in developed countries. It is proven how difficult it is to measure the efficacy of various treatment modalities for preterm labour. As the exact mechanism of preterm labour is not known efficacies can be assessed only in terms of subsequent clinical improvement rather than any biochemical or physiologic parameter. No progress had been made over the last two decades in reducing the incidence of preterm birth but some benefits have been identified from prolongation of pregnancy by enabling corticosteroid administration to accelerate fetal lung maturation, and the ability to transfer the pregnant women to a centre with improved neonatal intensive care unit facilities. A wide range of tocolytic agents has been used to inhibit preterm labour in order to have time for such cointervention. Terbutaline is one of the most widely used tocolytic drug in our setup.

Our findings are consistent with previous studies that have shown terbutaline as effective tocolytic agent accompanied by fewer side effects. ${ }^{12-14}$ These studies show that beta-adrenergic agonists are more effective compared to calcium channel blockers however, their significant side effects have limited their use in practice. On the other hand, these agents do not decrease the neonatal mortality and morbidity associated with preterm labour. ${ }^{12-15}$ Nevertheless, these agents are recommended as first-line tocolytic agents because of their efficacy. ${ }^{14,15}$

Mawaldi et al compared the effects of subcutaneous terbutaline with those of oral nifedipine for prolongation of gestation. ${ }^{12}$ Terbutaline and nifedipine appeared to be equally effective in their tocolytic action. It was also shown by Vani et al that oral terbutaline was more effective in preventing preterm labour until 37 weeks of gestation, as demonstrated by a lesser number of relapses during this period. ${ }^{17}$ The oral form of terbutaline was used as maintenance therapy in this study because it does not require intensive medical nursing care and observations and is not associated with the discomfort of an intravenous line. The dosage used in this study is equivalent to that commonly used to inhibit preterm labor.

These literatures support the efficacy of terbutaline in delaying delivery for 24 to 48 hours. Such a delay may be of benefit by increasing the time to delivery, allowing additional time for the beneficial effects of adjunctive corticosteroid therapy.

\section{CONCLUSION}

Administration of terbutaline prolonged the labour without serious maternal complications and better neonatal outcome. These findings make terbutaline the drug of choice for prolongation of pregnancy in those women with preterm labour. However, successful reduction of perinatal morbidity and mortality associated with prematurity may require the implementation of effective risk identification and behavioural modification programs for the prevention of preterm labor; these in turn require both an improved understanding of the psychosocial risk factors, etiology, and mechanisms of preterm labor and programs for accurate identification of pregnant women at risk for premature labor and delivery. The early identification of at risk pregnant women with timely referral and good obstetrical care may help decrease the extreme prematurity ( $<32$ week) rate, thereby reducing the morbidity, mortality, and the expenses associated with prematurity. Since the use of terbutaline is a much effective way of management of preterm labour and far cheaper than the cost incurred in the management of preterm neonate, use of terbutaline should definitely be explored on a larger scale.

\section{ACKNOWLEDGEMENTS}

This study was conducted with an immense support and invaluable suggestions from Dr. L. Padma and Dr. Mohammed Raza.

Funding: No funding sources

Conflict of interest: None declared

Ethical approval: The study was approved by the Institutional Ethics Committee

\section{REFERENCES}

1. World Health Organization: Recommendations of the WHO consultation of methodology of reporting and analysis of perinatal and maternal morbidity and mortality. Bristol; 1972.

2. Steer P. The epidemiology of preterm labor. BJOG. 2005;112(1):1-3.

3. Houtzager BA, Hogendoorn SM, Papatsonis DN, Samsom JF, van Geijn HP, Bleker OP, et al. Long term follow up of children exposed in utero to nifedipine or ritodrine for the management of preterm labor. BJOG. 2006;113:324-31.

4. Uma S, Nisha S, Shikha S. A prospective analysis of etiology and outcome of preterm labor. J Obstet Gynaecol India. 2007;57(1):48-52.

5. Crowley P. Prophylactic corticosteroids for preterm birth (Cochrane Review). Cochrane Database Syst Rev. 2000;(2):CD000065.

6. Keirse M. The history of tocolysis. BJOG. 2003;110(20):94-7.

7. Clinical green top guidelines: tocolytic drugs for women in preterm labour (1B) London UK. RCOG; 2002. 
8. Groome LJ, Goldenberg RL, Cliver SP, Davis RO, Copper RL. Neonatal periventricular-intra-ventricular hemorrhage after maternal beta-sympathominmetic tocolysis: The march of dimes multicenter study group. Am J Obstet Gynecol. 1992;167:873-9.

9. Treatment of preterm labor with the beta-adrenergic antagonist ritodrine: The Canadian preterm labor investigators group. N Engl J Med. 1992;327:308-12.

10. Gyetvai K, Hannah ME, Hodnett ED, Ohlsson A. Tocolytics for preterm labor: a systematic review. Obstet Gynecol. 1999;94:869-77.

11. Rodts-Palenik S, Morrison JC. Tocolysis: an update for the practitioner. Obstet Gynecol Surv. 2002;57:S9-34.

12. Mawaldi L, Duminy P, Tamim H. Terbutaline versus nifedipine for prolongation of pregnancy in patients with preterm labor. Int $\mathbf{J}$ Gynaecol Obstet. 2008; 100:65-8.
13. Collaris R, Tan PC. Oral nifepidine versus subcutaneous terbutaline tocolysis for external cephalic version: a double-blind randomised trial. BJOG. 2009;116:74-80.

14. Mohamed Ismail NA, Ibrahim M, Mohd Naim N, Mahdy ZA, Jamil MA, Mohd Razi ZR. Nifedipine versus terbutaline for tocolysis in external cephalic version. Int J Gynaecol Obstet. 2008;102:263-6.

15. Phupong V, Charakorn C, Charoenvidhya D. Oral salbutamol for treatment of preterm labor. J Med Assoc Thai. 2004;87:1012-6.

16. Trabelsi K, Hadj Taib H, Amouri H, Abdennadheur W, Ben Amar H, Kallel W, et al. Nicardipine versus salbutamol in the treatment of premature labor: Comparison of their efficacy and side effects. Tunis Med. 2008;86:43-8.

17. Vani S, Lau SY, Lim BK, Omar SZ, Tan PC. Intravenous salbutamol for external cephalic version. Int J Gynaecol Obstet. 2009;104:28-31.

Cite this article as: Priyadarshini BG, Kumar PR, Kumar TN. Efficacy of terbutaline in preterm labour - our experience. Int J Basic Clin Pharmacol 2016;5:2118-2122. 